\title{
ASSESSING THE EFFECTS OF LOGGING ACTIVITIES ON AVIAN RICHNESS AND DIVERSITY IN DIFFERENT AGED POST-HARVESTED HILL DIPTEROCARP TROPICAL RAINFOREST OF MALAYSIA
}

\author{
Rajpar, M.N. and M. Zakaria \\ Department of Recreation and Ecotourism, Faculty of Forestry, Universiti Putra Malaysia, \\ 43400 UPM Serdang, Selangor Darul Ehsan, Malaysia
}

Received 2014-01-23; Revised 2014-04-30; Accepted 2014-07-15

\begin{abstract}
Logging activities have encroached into the hill dipterocarp tropical rainforest area since the lowland dipterocarp forests have decreased in size. Hill dipterocarp tropical rainforest is rich in habitat diversity and provide a variety of resources for avian species such as food, habitat and shelter. Therefore it is important to examine the logging effects of hill dipterocarp rainforest on avian species. We compared the avian richness and diversity in different aged post-harvested hill dipterocarp tropical rainforest at the Berkelah Hill Dipterocarp Rainforest Reserve in Maran, Pahang, West Malaysia using mist-netting method. We captured a total of 1908 individuals representing 86 species and 29 families (i.e., $18.55 \%$ from two years postharvested forest, $25.10 \%$ from ten years post-harvested, $23.90 \%$ from twenty years post-harvested and $32.44 \%$ from thirty five years post-harvested forests). Forty nine species were caught in two years and ten years, 55 species in twenty years and 59 species in thirty five years' post-harvested forest. Seventeen species were common in all four types of forest. Pycnonotidae, Timaliidae and Nectariniidae were the most dominant families in all types of post-harvested hill dipterocarp tropical rainforest. Diversity analysis indicated that the bird species in twenty years post-harvested hill dipterocarp rainforest was most diverse (i.e., Fisher's Alpha Diversity Index; 16.34) and evenly distributed (i.e., McIntosh Evenness index E; 0.933) as compared to two years, ten years and thirty five years post-harvested forest. However, thirty five years post-harvested forest was richest in avian species (i.e., Margalef's Richness index $\mathrm{R}_{1}$; 9.02) as compared to other post-harvested forest. The findings of this study revealed that logging and recovery process may affects on avian distribution and diversity. However, these effects may vary from species to species.
\end{abstract}

Keywords: Birds, Rainforest, Mist-Netting, Post-Harvesting, Species Richness

\section{INTRODUCTION}

Malaysia is blessed with a variety of forest types such as lowland dipterocarp forest, hill dipterocarp forest, upper-hill dipterocarp forest, oak-laurel forest, montane ericaceous forest, peat swamp forest, mangrove forests. Malaysian hill forests are dominated by trees of dipterocarpaceae which grows from $300 \mathrm{~m}$ to an altitude of $900 \mathrm{~m}$. The significant feature of hill dipterocarp forest is the presence of Seraya
(Shorea sp.) large size trees which most frequently grows on hill ridges with prolific undergrowth of Eugeisonna tristis (Bertam palm) and Oncosperma horridum (Thorny palm tree). These forests are the most diverse in vegetation structure and composition that supported a diversity of wildlife species especially avian which directly or indirectly depend on the forests for survival.

Malaysia is rich in vegetation diversity and are home of 742 bird species which directly or indirectly use the forest 43400 UPM Serdang, Selangor Darul Ehsan, Malaysia 
habitats. Despite of rich in avian diversity, 50\% of tropical forests have been destroyed and degraded at alarming rate due to anthropogenic activities such as urbanization, conversion into agricultural fields (Fitzherbert et al., 2008; Edwards et al., 2011; Fisher et al., 2011) and logging activities (Fahrig, 2003; Castelletta et al., 2005; 2000; Clark et al., 2009). It has been reported that protected forest areas of Southeast Asia has been fragmented and reduced due to deforestation (Laurance, 1999; Curran et al., 2004; DeFries et al., 2005).

Forest logging may results in habitat fragmentation which can lead to reductions in nutrient availability and habitat for a wide array of wildlife species (Chaves et al., 2012). Forest logging directly or indirectly reduced the habitat suitability of forest fauna (Potts, 2011) such as butterflies and mammals (Brook et al., 2003; Sehgal, 2010) and monkeys (Collins, 2008). Due to logging, the forest becomes fragmented, more irregular and isolated (Echeverria et al., 2007) which affects on distribution, richness and diversity of avian species (McCarthy, 2012). In addition, logging also modified plant species diversity, vegetation composition and structure (Hill and Curran, 2003; Arroyo-Rodriguez and Mandujano, 2006) that may alter or reduced food resources and habitat suitability (Arroyo-Rodriguez and Mandujano, 2006). It has been assessed that the forest area loss and degradation has seriously affected the community structures of wildlife species i.e., some of them become extinct while others are endangered and vulnerable due to habitat loss and degradation (Koh et al., 2004; Cardillo et al., 2005; Brook et al., 2006; Sodhi and Brook, 2006).

Currently, the information regarding the effects of hill dipterocarp tropical forest logging on wildlife species is not sufficient and it needed more investigation. Hence, it is extremely important to study the avian richness and diversity in different aged post-harvested hill dipterocarp rainforest in order to understand the effects of logging and recovery process on avian community for future direction and conservation activities. In this study, we examine the avian richness and diversity in two years, ten years, twenty years and thirty years post-harvested forests.

\section{MATERIALS AND METHODS}

\subsection{Study Area}

This study is located at the Berkelah Hill Dipterocarp Rainforest Reserve in Maran, Pahang, West Malaysia ( $2^{\circ} 57^{\prime} 43^{\prime}$ N, $101^{\circ} 41^{\prime} 47^{\prime}$ ' E) (Fig. 1). This hill dipterocarp rainforest consists of mixture of undisturbed primary forest and different aged postharvested forests. We selected two years, ten years, twenty years and thirty five years post-harvested forest areas within the forest reserve.

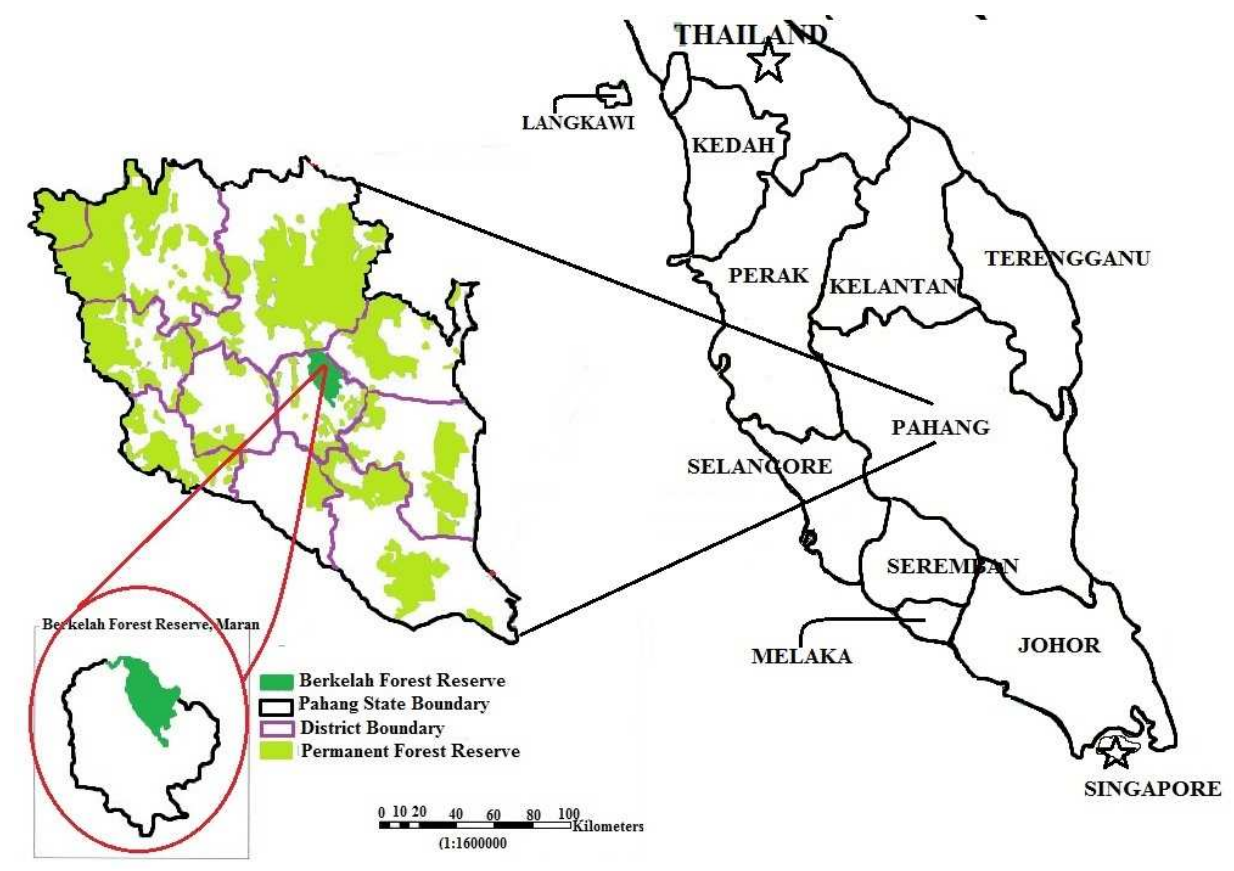

Fig. 1. Location map of Berkelah forest reserve Maran, Pahang, West Malaysia 


\subsection{Bird Surveys}

Bird species were caught using ten mist-nets $(14 \times 4$ $\mathrm{m}$ with 3 pockets) in two years, ten years, twenty years and thirty years post-harvested forests. The netting was done for a total of $3,084 \mathrm{~h}$ or 257 days from Janury 2011 to December, 2012. The nets were stretched between two bamboo poles that were fixed into soil. The lower end of the net was kept at the ground to capture all type of birds at different locations. The nets were opened at 0700-1900 $\mathrm{h}$ and placed for three days in the same sampling site before transferred to new site. Three days netting was sufficient to capture most of the birds as after three days, birds may become familiar with the mist nets (Robbins et al., 1997). The nets were monitored hourly and each individual bird captured was tagged with a numbered aluminium ring on the right tarsus and photographed before they were released (Robson, 2002; Ralph and Dunn, 2004; Rajpar and Zakaria, 2010; 2012).

\section{DATA ANALYSIS}

\subsection{Relative Abundance (\%)}

Relative abundance refers to the number of individuals of a particular species as percent of the total capture in both areas. We estimated relative abundances for each species using average detection values calculated by dividing the total number of a species captured at different aged post-harvested hill dipterocarp tropical rainforest. The relative abundance $(\%)$ of bird species was estimated using the following expression:

Relative abundance $=n / N \times 100$

Where:

$\mathrm{n}=$ The number of a particular captured bird species while $\mathrm{N}=$ The total number captured over all species

\subsection{Bird Diversity Indices}

Avian species diversity, species richness and species evenness in different aged post-harvested hill dipterocarp tropical rainforests were analyzed using Community Analysis Package Software (CAP, Version 4.0) (Henderson and Seaby, 2007).

The Fisher's alpha for each forest type was calculated using the following equation:

$$
S=a * \ln (l+n / a)
$$

Where:
$\mathrm{S}=$ Number of taxa

$\mathrm{n}=$ Number of individuals and

$\mathrm{a}=$ Fisher's alpha

Margalef's richness index was calculated using the following equation:

$$
R_{1}=(S-l) / \ln (n)
$$

Where:

$\mathrm{S}=$ Number of taxa and

$\mathrm{N}=$ Number of individuals

McIntosh evenness index E was calculated using the following equation:

$$
M c E=\left[N-\sqrt{ }\left(\sum n i 2\right] /[N-(N / \sqrt{ } S)]\right.
$$

Where:

$\mathrm{McE}=$ McIntosh evenness index

$\mathrm{n}_{\mathrm{i}} \quad=$ Number of individuals belonging to i species,

$\mathrm{S} \quad=$ Total number of species and

$\mathrm{N} \quad=$ Total number of individuals

\subsection{Significant Difference Among Different Aged Post-Harvested Forest Habitats}

A One-Way Analysis of Variance (ANOVA) and Tukey's (HSD) test (Analytical Software, version 8.1) by (McGraw-Hill, 2008) was conducted in order to investigate the difference in bird richness and habitat characteristics between primary and logged hill dipterocarp tropical rainforest.

\section{RESULTS}

\subsection{Bird Species Composition with Relative Abundance in Different Aged Post-Harvested Hill Dipterocarp Tropical Rainforests}

Overall, mist-netting method captured a total of 1908 bird individuals representing 86 bird species and 29 families (i.e., $18.55 \%$ from two years postharvested forest, $25.10 \%$ from ten years postharvested, $23.90 \%$ from twenty years post-harvested and $32.44 \%$ from thirty five years' post-harvested forests). Seventeen bird species were captured from all types of forest while 49 bird species (each) were caught in two years and ten years post-harvested forest, 55 bird species were caught in twenty years post-harvested forest and 59 bird species were caught in thirty five years post-harvested forest. 


\subsection{Bird Species Composition with Relative Abundance in Two Years Post-Harvested Forest}

A total of 354 bird individuals were captured (i.e., $18.55 \%$ ) from two years post-harvested hill dipterocarp rainforest representing 49 bird species and 21 families. Three bird species i.e., Arachnothera longirostra-Little Spiderhunter $(4.09 \%)$, Pycnonotus simplex-Creamvented Bulbul and P. erythropthalmo-Spectacled Bulbul (1.15\% each) were the most common bird species in the two years post-harvested forest. On the contrarily, four bird species i.e., Ixos malaccensis-Streaked Bulbul, Copsychus malabaricus-White-rumped Shama, Harpactes diardii-Diard's Trogon and Eurylaimus javanicus-Banded Broadbill were least abundant (each $0.05 \%$ - captured only once; Table $\mathbf{1})$.

\subsection{Bird Species Composition with Relative Abundance in Ten Years Post-Harvested Forest}

In the ten years post-harvested hill dipterocarp rainforest a total of 479 bird individuals of 49 species representing 18 families were captured. The results indicated that A. longirostra-Little Spiderhunter (4.93\%), Alophoixus phaeocephalus-Yellow-bellied Bulbul (1.26\%) and Hypogramma hypogrammicum-Purplenaped Sunbird $(1.10 \%)$ were the most abundant bird species in ten years post-harvested forest. In contrast, four species namely Pitta guajan-Banded Pitta, Picus miniaceus-Banded Woodpecker, Platylophus galericulatus-Crested Jay and Batrachostomus stellatusGould's Frogmouth were considered as the rarest (each $0.05 \%$ ) (Table 1).

\subsection{Bird Species Composition with Relative Abundance in Twenty Years Post-Harvested Forest}

Likewise, a total of 456 bird individuals belonging to 55 species and 21 families were captured from twenty years post-harvested hill dipterocarp rainforest. Four bird species namely; A. longirostra-Little Spiderhunter $(2.25 \%), \quad H$. hypogrammicum-Purple-naped Sunbird (1.42\%), Meiglyptes tukki-Buff-necked Woodpecker (1.26\%) and Malacopteron magnum-Rufous-crowned Babbler $(1.15 \%)$ were the most abundant bird species captured with higher number of individuals. In addition, 13 bird species such as Malacopteron cinereum-Scalycrowned Babbler, Pycnonotus bruuneus-Redeye Bulbul, Iole olivacea-Buff-vented Bulbul, P. cyaniventris-Grey- bellied Bulbul, Chloropsis cyanopogon-Lesser Green Leafbird, Lonchura leucogastra-White-bellied Munia, Malacocincla malaccensis-Short-tailed Babbler, $B$. stellatus-Gould's Frogmouth, Anthreptes simplexPlain Sunbird, Calormphus fuliginosus-Brown Barbet, Prionochilus percussus-Crimson-breasted Flowerpecker, A. magna-Streaked Spiderhunter and Eumyias thalassinus-Verditer Flycatcher were considered as the rarest bird species in twenty year postharvested forest i.e., (i.e., $0.05 \%$ each) (Table 1).

\subsection{Bird Species Composition with Relative Abundance in Thirty Five Years Post- Harvested Forest}

A total of 619 bird individuals belong to 59 bird species and 21 families were caught from thirty five year post-harvested hill dipterocarp rainforest. The results indicate that $M$. magnum-Rufous-crowned Babbler (2.88\%), Malacopteron magnirostre-Moustached Babbler (1.83\%), H. hypogrammicum-Purple-naped Sunbird (1.78\%), A. phaeocephalus-Yellow-bellied Bulbul (1.68\%) and Meiglyptes tukki-Buff-necked Woodpecker $(1.62 \%)$ were the most dominant bird species in thirty years post-harvested hill dipterocarp rainforest. On contrary, eight bird species i.e., Trichastoma rostratum-White-chested Babbler, $P$. miniaceus-Banded Woodpecker, A. simplex-Plain Sunbird, Lacedo pulchella-Banded Kingfisher, Enicurus ruficapillus-Chestnut-naped Forktail, Muscicapella hodgsoni-Pygmy Blue Flycatcher, Otus rufescensReddish Scope Owl and Ficedula zanthopygia-Yellowrumped Flycatcher were the rarest (i.e., $0.05 \%$ each) in thirty year years post-harvested forest (Table 1).

\subsection{Comparison of Bird Species Composition in Different Aged Post-Harvested Forests}

Twenty seven bird species were commonly captured from two years and twenty years post-harvested forest, However, twenty two bird species were sampled only in a two years post-harvested forest which were absent in ten years post-harvested forest. Likewise, twenty two bird species were captured in ten years post-harvested forest but absent in a two years post-harvested forest. In addition, 29 bird species were commonly detected in two years post harvested and twenty years post-harvested forest. However, 20 bird species were recorded only in a two year post harvested forest which were absent in twenty years postharvested forest. Similarly, 26 bird species were captured only in twenty years post-harvested forest and were absent in a two year post-harvested forest (Table 1). 
Rajpar, M.N. and M. Zakaria / American Journal of Applied Sciences 11 (9): 1519-1529, 2014

Table 1. Bird species composition with relative abundance in different aged post-harvested hill dipterocarp tropical rainforest at Berkelah Forest Reserve Maran, Pahang, West Malaysia

\begin{tabular}{|c|c|c|c|c|c|c|c|c|c|c|}
\hline \multirow[b]{2}{*}{ Family } & \multirow[b]{2}{*}{ Scientific name } & \multirow[b]{2}{*}{ Common name } & \multicolumn{2}{|c|}{$\begin{array}{l}\text { Two years } \\
\text { post-harvested } \\
\text { forest }\end{array}$} & \multicolumn{2}{|c|}{$\begin{array}{l}\text { Ten years } \\
\text { post-harvested } \\
\text { forest }\end{array}$} & \multicolumn{2}{|c|}{$\begin{array}{l}\text { Ten years } \\
\text { post-harvested } \\
\text { forest }\end{array}$} & \multicolumn{2}{|c|}{$\begin{array}{l}\text { Thirty five years } \\
\text { post-harvested } \\
\text { forest }\end{array}$} \\
\hline & & & $\begin{array}{l}\text { No of } \\
\text { captures }\end{array}$ & $\%$ & $\begin{array}{l}\text { No of } \\
\text { captures }\end{array}$ & $\%$ & $\begin{array}{l}\text { No of } \\
\text { captures }\end{array}$ & $\%$ & $\begin{array}{l}\text { No of } \\
\text { captures }\end{array}$ & $\%$ \\
\hline Nectariniidae & Arachnothera longirostra & Little Spiderhunter & 78 & 4.09 & 94 & 4.93 & 43 & 2.25 & 28 & 1.47 \\
\hline Pycnonotidae & Pycnonotus simplex & Cream-vented Bulbul & 22 & 1.15 & 16 & 0.84 & 12 & 0.63 & 9 & 0.47 \\
\hline Pycnonotidae & Pycnonotus erythropthalmos & Spectacled Bulbul & 22 & 1.15 & 14 & 0.73 & 11 & 0.58 & 8 & 0.42 \\
\hline Meropidae & Merops viridis & Blue-throated Bee-eater & 19 & 0.84 & 14 & 0.73 & 11 & 0.58 & 3 & 0.16 \\
\hline Timaliidae & Malacopteron cinereum & Scaly-crowned Babbler & 16 & 0.84 & 17 & 0.89 & 1 & 0.05 & 0 & 0.00 \\
\hline Pycnonotidae & Pycnonotus bruuneus & Redeye Bulbul & 13 & 0.68 & 9 & 0.47 & 1 & 0.05 & 3 & 0.16 \\
\hline Timaliidae & Malacopteron magnum & Rufous-crowned Babbler & 11 & 0.58 & 17 & 0.89 & 22 & 1.15 & 55 & 2.88 \\
\hline Nectariniidae & Archnothera modesta & Grey-breasted Spiderhunter & 11 & 0.58 & 8 & 0.42 & 7 & 0.37 & 12 & 0.63 \\
\hline Turdidae & Copsychus saularis & Oriental Magpie Robin & 9 & 0.47 & 6 & 0.31 & 4 & 0.21 & 0 & 0.00 \\
\hline Alcedinidae & Alcedo meninting & Blue-eared Kingfisher & 9 & 0.47 & 4 & 0.21 & 3 & 0.16 & 2 & 0.10 \\
\hline Cisticolidae & Orthotomus atrogularis & Dark-necked Tailorbird & 9 & 0.47 & 0 & 0.00 & 0 & 0.00 & 0 & 0.00 \\
\hline Eurylaimidae & Cymbirhynchus macrorhynchos & Black-and-red Broadbill & 8 & 0.42 & 5 & 0.26 & 2 & 0.10 & 0 & 0.00 \\
\hline Cuculidae & Cacomantis merulinus & Plaintive Cuckoo & 8 & 0.42 & 5 & 0.26 & 00 & 0.00 & 0 & 0.00 \\
\hline Picidae & Meiglyptes tukki & Buff-necked Woodpecker & 7 & 0.37 & 19 & 1.00 & 24 & 1.26 & 31 & 1.62 \\
\hline Laniidae & Lanius cristatus & Brown Shrike & 7 & 0.37 & 0 & 0.00 & 00 & 0.00 & 0 & 0.00 \\
\hline Timaliidae & Stachyris erythroptera & Chestnut-winged Babbler & 6 & 0.31 & 9 & 0.47 & 12 & 0.63 & 19 & 1.00 \\
\hline Pycnonotidae & Pycnonotus finalysoni & Stripe-throated Bulbul & 6 & 0.31 & 4 & 0.21 & 2 & 0.10 & 0 & 0.00 \\
\hline Hirundinidae & Hirundo rustica & Barn Swallow & 6 & 0.31 & 0 & 0.00 & 0 & 0.00 & 0 & 0.00 \\
\hline Nectariniidae & Arachnothera flavigaster & Spectacled Spiderhunter & 6 & 0.31 & 0 & 0.00 & 0 & 0.00 & 0 & 0.00 \\
\hline Aegithinidae & Aegithina viridissima & Green Iora & 5 & 0.26 & 0 & 0.00 & 0 & 0.00 & 0 & 0.00 \\
\hline Laniidae & Lanius tigrinus & Tiger Shrike & 5 & 0.26 & 0 & 0.00 & 0 & 0.00 & 0 & 0.00 \\
\hline Pycnonotidae & Alophoixus phaeocephalus & Yellow-bellied Bulbul & 4 & 0.21 & 24 & 1.26 & 19 & 1.00 & 32 & 1.68 \\
\hline Turdidae & Luscinia cyne & Siberian Blue Robin & 4 & 0.21 & 15 & 0.79 & 7 & 0.37 & 11 & 0.58 \\
\hline Muscicapidae & Philentoma pyrhoptera & Rufous-winged Philentoma & 4 & 0.21 & 9 & 0.47 & 14 & 0.73 & 17 & 0.89 \\
\hline Pycnonotidae & Tricholestes criniger & Hairy-backed Bulbul & 4 & 0.21 & 9 & 0.47 & 16 & 0.84 & 24 & 1.26 \\
\hline Pycnonotidae & Pycnonotus eutilotus & Puff-backed Bulbul & 4 & 0.21 & 2 & 0.10 & 0 & 0.00 & 0 & 0.00 \\
\hline Oriolidae & Oriolus xanthonotus & Dark-throated Oriole & 4 & 0.21 & 0 & 0.00 & 2 & 0.10 & 2 & 0.10 \\
\hline Muscicapidae & Muscicapa dauurica & Asian Brown Flycatcher & 4 & 0.21 & 0 & 0.00 & 0 & 0.00 & 0 & 0.00 \\
\hline Campephagidae & Hemipus hirundinaceus & Black-winged Flycatcher Shrike & 4 & 0.21 & 0 & 0.00 & 0 & 0.00 & 0 & 0.00 \\
\hline Timaliidae & Trichastoma rostratum & White-chested Babbler & 3 & 0.16 & 7 & 0.37 & 0 & 0.00 & 1 & 0.05 \\
\hline Timaliidae & Macronus ptilosus & Fluffy-backed Tit-Babbler & 3 & 0.16 & 2 & 0.10 & 0 & 0.00 & 0 & 0.00 \\
\hline Chloropseidae & Chloropsis cochinchinensis & Blue-winged Leafbird & 3 & 0.16 & 0 & 0.00 & 0 & 0.00 & 0 & 0.00 \\
\hline Pycnonotidae & Alophoixus ochraceus & Ochraceous Bulbul & 2 & 0.10 & 11 & 0.58 & 16 & 0.84 & 19 & 1.00 \\
\hline Eurylaimidae & Calyptomena viridis & Green Broadbill & 2 & 0.10 & 10 & 0.52 & 14 & 0.73 & 21 & 1.10 \\
\hline Pycnonotidae & Pycnonotus plumosus & Olive-winged Bulbul & 2 & 0.10 & 0 & 0.00 & 6 & 0.31 & 0 & 0.00 \\
\hline Cisticolidae & Prinia rufescens & Rufescent Prinia & 2 & 0.10 & 0 & 0.00 & 2 & 0.10 & 0 & 0.00 \\
\hline Pycnonotidae & Iole olivacea & Buff-vented Bulbul & 2 & 0.10 & 0 & 0.00 & 1 & 0.05 & 0 & 0.00 \\
\hline Pycnonotidae & Pycnonotus cyaniventris & Grey-bellied Bulbul & 2 & 0.10 & 0 & 0.00 & 1 & 0.05 & 0 & 0.00 \\
\hline Chloropseidae & Chloropsis cyanopogon & Lesser Green Leafbird & 2 & 0.10 & 0 & 0.00 & 1 & 0.05 & 0 & 0.00 \\
\hline Estrildidae & Lonchura leucogastra & White-bellied Munia & 2 & 0.10 & 0 & 0.00 & 1 & 0.05 & 0 & 0.00 \\
\hline Falconidae & Microhierax fringillarius & Black-thighed Falconet & 2 & 0.10 & 0 & 0.00 & 0 & 0.00 & 0 & 0.00 \\
\hline Timaliidae & Malaconcincla sepiaria & Horsfield's Babbler & 2 & 0.10 & 0 & 0.00 & 0 & 0.00 & 2 & 0.10 \\
\hline Muscicapidae & Ficedula mugimaki & Mugimaki Flycatcher & 2 & 0.10 & 0 & 0.00 & 0 & 0.00 & 0 & 0.00 \\
\hline Dicaeidae & Dicaeum trignostigma & Orange-bellied Flowerpecker & 2 & 0.10 & 0 & 0.00 & 0 & 0.00 & 0 & 0.00 \\
\hline Timaliidae & Macronus gularis & Pin-striped Tit-Babbler & 2 & 0.10 & 0 & 0.00 & 0 & 0.00 & 0 & 0.00 \\
\hline Pycnonotidae & Ixos malaccensis & Streaked Bulbul & 1 & 0.05 & 9 & 0.47 & 14 & 0.73 & 19 & 1.00 \\
\hline Muscicapidae & Copsychus malabaricus & White-rumped Shama & 1 & 0.05 & 8 & 0.42 & 17 & 0.89 & 22 & 1.15 \\
\hline Trogonidae & Harpactes diardii & Diard's Trogon & 1 & 0.05 & 2 & 0.10 & 0 & 0.00 & 0 & 0.00 \\
\hline Eurylaimidae & Eurylaimus javanicus & Banded Broadbill & 1 & 0.05 & 0 & 0.00 & 0 & 0.00 & 4 & 0.21 \\
\hline Nectariniidae & Hypogramma hypogrammicum & Purple-naped Sunbird & 0 & 0.00 & 21 & 1.10 & 27 & 1.42 & 34 & 1.78 \\
\hline Timaliidae & Malacopteron magnirostre & Moustached Babbler & 0 & 0.00 & 16 & 0.84 & 23 & 1.21 & 35 & 1.83 \\
\hline Alcedinidae & Ceyx rufidorsa & Rufous-backed Kingfisher & 0 & 0.00 & 11 & 0.58 & 15 & 0.79 & 20 & 1.05 \\
\hline Monarchidae & Terpsiphone paradisi & Asian Paradise Flycatcher & 0 & 0.00 & 9 & 0.47 & 8 & 0.42 & 11 & 0.58 \\
\hline Timaliidae & Stachyris poliocephala & Grey-headed Babbler & 0 & 0.00 & 9 & 0.47 & 18 & 0.94 & 28 & 1.47 \\
\hline Alcedinidae & Alcedo euryzona & Blue-banded Kingfisher & 0 & 0.00 & 7 & 0.37 & 0 & 0.00 & 2 & 0.10 \\
\hline Irenidae & Irena puella & Asian Fairy-bluebird & 0 & 0.00 & 6 & 0.31 & 2 & 0.10 & 2 & 0.10 \\
\hline Timaliidae & Malacocincla malaccensis & Short-tailed Babbler & 0 & 0.00 & 6 & 0.31 & 1 & 0.05 & 3 & 0.16 \\
\hline Timaliidae & Stachyris nigricollis & Black-throated Babbler & 0 & 0.00 & 6 & 0.31 & 10 & 0.52 & 13 & 0.68 \\
\hline Rhipuduridae & Rhipidura perlata & Spotted Fantail & 0 & 0.00 & 6 & 0.31 & 3 & 0.16 & 5 & 0.26 \\
\hline Monarchidae & Hypothymis azurea & Black-naped Monarch & 0 & 0.00 & 5 & 0.26 & 8 & 0.42 & 13 & 0.68 \\
\hline
\end{tabular}


Table 1. Continue

\begin{tabular}{|c|c|c|c|c|c|c|c|c|c|c|}
\hline Timaliidae & Trichastoma bicolor & Ferruginous Babbler & 0 & 0.00 & 5 & 0.26 & 6 & 0.31 & 10 & 0.52 \\
\hline Muscicapidae & Rhinomyias umbratilis & Grey-chested Jungle Flycatcher & 0 & 0.00 & 5 & 0.26 & 12 & 0.63 & 16 & 0.84 \\
\hline Timaliidae & Pellorneum capistratum & Black-capped Babbler & 0 & 0.00 & 4 & 0.21 & 6 & 0.31 & 14 & 0.73 \\
\hline Picidae & Sasia abnormis & Rufous Piculet & 0 & 0.00 & 3 & 0.16 & 2 & 0.10 & 3 & 0.16 \\
\hline Picidae & Picus mentalis & Checker-throated Woodpecker & 0 & 0.00 & 3 & 0.16 & 6 & 0.31 & 8 & 0.42 \\
\hline Alcedinidae & Alcedo atthis & Common Kingfisher & 0 & 0.00 & 2 & 0.10 & 4 & 0.21 & 7 & 0.37 \\
\hline Picidae & Blythipicus rubiginosus & Maroon Woodpecker & 0 & 0.00 & 2 & 0.10 & 3 & 0.16 & 4 & 0.21 \\
\hline Pittidae & Pitta guajana & Banded Pitta & 0 & 0.00 & 1 & 0.05 & 2 & 0.10 & 3 & 0.16 \\
\hline Picidae & Picus miniaceus & Banded Woodpecker & 0 & 0.00 & 1 & 0.05 & 0 & 0.00 & 1 & 0.05 \\
\hline Corvidae & Platylophus galericulatus & Crested Jay & 0 & 0.00 & 1 & 0.05 & 3 & 0.16 & 5 & 0.26 \\
\hline Podargidae & Batrachostomus stellatus & Gould's Frogmouth & 0 & 0.00 & 1 & 0.05 & 1 & 0.05 & 3 & 0.16 \\
\hline Alcedinidae & Ceyx erithaca & Black-backed Kingfisher & 0 & 0.00 & 0 & 0.00 & 5 & 0.26 & 6 & 0.31 \\
\hline Nectariniidae & Anthreptes simplex & Plain Sunbird & 0 & 0.00 & 0 & 0.00 & 1 & 0.05 & 1 & 0.05 \\
\hline Alcedinidae & Lacedo pulchella & Banded Kingfisher & 0 & 0.00 & 0 & 0.00 & 0 & 0.00 & 1 & 0.05 \\
\hline Pycnonotidae & Pycnonotus melanoleucos & Black-and-white Bulbul & 0 & 0.00 & 0 & 0.00 & 0 & 0.00 & 2 & 0.10 \\
\hline Ramphastidae & Calormphus fuliginosus & Brown Barbet & 0 & 0.00 & 0 & 0.00 & 1 & 0.05 & 2 & 0.10 \\
\hline Muscicapidae & Enicurus ruficapillus & Chestnut-naped Forktail & 0 & 0.00 & 0 & 0.00 & 0 & 0.00 & 1 & 0.05 \\
\hline Timaliidae & Stachyris maculata & Chestnut-rumped Babbler & 0 & 0.00 & 0 & 0.00 & 0 & 0.00 & 8 & 0.42 \\
\hline Trogonidae & Harpactes orrhophaeus & Cinnamon-rumped Trogon & 0 & 0.00 & 0 & 0.00 & 0 & 0.00 & 2 & 0.10 \\
\hline Dicaeidae & Prionochilus percussus & Crimson-breasted Flowerpecker & 0 & 0.00 & 0 & 0.00 & 1 & 0.03 & 0 & 16.00 \\
\hline Muscicapidae & Muscicapella hodgsoni & Pygmy Blue Flycatcher & 0 & 0.00 & 0 & 0.00 & 0 & 0.00 & 1 & 0.05 \\
\hline Strigidae & Otus rufescens & Reddish Scope Owl & 0 & 0.00 & 0 & 0.00 & 0 & 0.00 & 1 & 0.05 \\
\hline Timaliidae & Malacopteron affine & Sooty-capped Babbler & 0 & 0.00 & 0 & 0.00 & 0 & 0.00 & 2 & 0.10 \\
\hline Nectariniidae & Arachnothera magna & Streaked Spiderhunter & 0 & 0.00 & 0 & 0.00 & 1 & 0.05 & 2 & 0.10 \\
\hline Muscicapidae & Eumyias thalassinus & Verditer Flycatcher & 0 & 0.00 & 0 & 0.00 & 1 & 0.05 & 2 & 0.10 \\
\hline \multirow[t]{2}{*}{ Muscicapidae } & Ficedula zanthopygia & Yellow-rumped Flycatcher & 0 & 0.00 & 0 & 0.00 & 0 & 0.00 & 1 & 0.05 \\
\hline & & Total & 354 & & 479 & & 456 & & 619 & \\
\hline
\end{tabular}

Table 2. Diversity indices of bird species in different post-harvested hill dipterocarp tropical rainforest at Berkelah Reserve Maran, Pahang, West Malaysia

\begin{tabular}{|c|c|c|c|}
\hline Habitat & $\begin{array}{l}\text { Fisher's alpha } \\
\text { diversity index }\end{array}$ & $\begin{array}{l}\text { Margalef's } \\
\text { richness index } \mathrm{R}_{1}\end{array}$ & $\begin{array}{l}\text { McIntosh } \\
\text { evenness index E }\end{array}$ \\
\hline Two years post-harvested hill & 15.43 & 8.18 & 0.855 \\
\hline $\begin{array}{l}\text { Dipterocarp tropical rainforest } \\
\text { Ten years post-harvested hill } \\
\text { Dipterocarp tropical rainforest }\end{array}$ & 13.66 & 7.78 & 0.884 \\
\hline $\begin{array}{l}\text { Twenty years post-harvested hill } \\
\text { Dipterocarp tropical rainforest }\end{array}$ & 16.34 & 8.82 & 0.933 \\
\hline $\begin{array}{l}\text { Thirty five years post-harvested hill } \\
\text { Dipterocarp tropical rainforest }\end{array}$ & 16.02 & 9.02 & 0.929 \\
\hline
\end{tabular}

The comparison results of two year post-harvested forest and thirty five year post-harvested forest indicated that 22 bird species were common, 27 bird species only used two year post-harvested forest and avoided to use thirty year post-harvested forest. Likewise, 37 bird species only utilized thirty five year post-harvested forest and totally avoided to visit two year post-harvested forest. The comparative results of ten year post-harvested and twenty years postharvested forest showed that 42 bird species were common which utilized both types of habitats and 7 bird species only prefer to utilize ten year postharvested forest and avoided twenty years postharvested forest. Similarly, 13 bird species only utilized twenty years post-harvested forest and avoided ten year post-harvested forest (Table 1).
In addition, the comparison results of twenty years and thirty five year post-harvested forest revealed that 45 bird species commonly utilized both types of post-harvested forests. However, 10 bird species only used twenty years post-harvested forest and were absent in thirty five year post-harvested forest. Likewise, 14 bird species only captured in thirty year post-harvested forest and were absent in twenty years post-harvested forest (Table 1).

Furthermore, the comparison results of the One-way ANOVA and Tukey's (HSD) test revealed that mean avian relative abundance of two years post-harvested hill dipterocarp rainforest $(4.12 \pm 1.07)$, ten years postharvested hill dipterocarp rainforest $(5.57 \pm 1,66)$, twenty years post-harvested hill dipterocarp rainforest $(5.30 \pm 1.51)$ and thirty five years post-harvested hill 
dipterocarp rainforest (7.20 \pm 1.47$)$ was not significantly different from each other $\left(\mathrm{F}_{3}, 343=1.42, \mathrm{p}<0.05\right)$.

\subsection{Comparison of Bird Diversity Indices in Different Aged Post-Harvested Forests}

The diversity analysis indicated that the bird species in the twenty years post-harvested hill dipterocarp rainforest was most diverse (i.e., Fisher's Alpha Diversity Index; 16.34) and most evenly distributed (i.e., McIntosh Evenness index E; 0.933) as compared to the two years, twenty years and thirty five years post-harvested forests. However, thirty years post-harvested forest was richest in avian species (i.e., Margalef's Richness index $\mathrm{R}_{1 \text {; }}$ 9.02) as compared to other post-harvested forest (Table 2).

\section{DISCUSSION}

The hill dipterocarp tropical rainforest is rich in habitat diversity that offers a variety of resources for avian species such as food, habitat and shelter. Birds are bio-indicators of forest ecosystem health i.e., they are more conspicuous, easy to study and are closely associated with vegetation structure. Birds may exploit different types of vegetated areas (Gill, 2006) and occupy a wide range of habitats. The recording of 49 bird species (each) from two years and ten years post-harvested hill dipterocarp tropical rainforest, 55 bird species from twenty years post-harvested forest and 59 bird species from thirty five years' postharvested hill dipterocarp tropical rainforest indicated that forest logging activities and recovery process may affects avian distribution, richness and diversity directly and indirectly.

This also indicated that the thirty five years postharvested hill dipterocarp forest has replaced the loss of vegetation and harboured a wide array of avian species richness and diversity as compared two years, ten years and twenty years post-harvested hill dipterocarp rainforest. It has been reported that many aspects of the forest especially the vegetation structure, composition and food resources have recovered (Chazdon et al., 2007; Dent and Wright, 2009) that may accommodate higher avian richness and diversity (Ellwood et al., 2002; Ellwood and Foster, 2004). Food resources distribution may regulate population of bird species (Wright et al., 1999; Johnson and Sherry, 2001) and fruit abundance influences the species composition and foraging behaviour of fruit eating birds (Moegenburg and Levey, 2003). For example; fruit eating birds always concentrated where fruit occurs abundantly because their diet consists of more than 50\% fruits (Kinnard et al., 1996; Sherman and Eason, 1998; Malizia, 2001; Renton, 2001).

The capturing of higher number of sunbirds, spider-hunters and bulbuls at the two years postharvested forest indicated that forest logging is a major determinant factors which effects on the richness and distribution of these bird species by modifying vegetation structure and composition (McShea and Rappole, 2000) and these bird species are resilience to disturbance. These bird species are considered as open country birds and utilized open area such as parks, gardens and plantations. (Campbell et al., 2007; Costello et al., 2000) reported that songbird species diversity increased in harvested areas i.e., they often prefers interior edge, forest gaps and logged areas (Moorman et al., 2002; Gram et al., 2003; King and DeGraaf, 2004). This might be that an open areas may provide suitable habitat and foraging sites for them (Gram et al., 2003; Campbell et al., 2007). Selective forest logging leads to the increase of temperature and decrease relative humidity (Johns, 1988; Jackson et al., 2002). Opening gaps enhance shrub vegetation which frequently bears diverse flowers and fruits which is a major diet of these bird species. (Doyon et al., 2005; Pers, 2000) and (Robinson and Robinson, 1999) stated that logging of trees cause gaps which enhance the growth of shrubs (understorey vegetation) which attract the understorey bird species such as warbler.

Likewise, capturing of good numbers of babbler species at the thirty five years post-harvested forest showed that these bird species are less resilient to disturbance and are habitat specialists. This indicated that bird richness and diversity increase with recovery after logging. Tree diversity and richness affect the food availability and accessibility that ultimately influence bird distribution and diversity. The variation in habitat selection might be due to differences in foraging behaviour and niches i.e., some inhabit canopy to hunt on flies or forage on fruits (barbets). The heterogeneity of vegetation affects productivity (Belisle et al., 2001; Ishikawa et al., 2003; Currie et al., 2004) that potentially offer more niches for avian species through providing a wide range of resources such as food, suitable shelter and nesting sites and also safe roosting sites. Avian richness is associated with the abundance, distribution and diversity of food resources (Marquez et al., 2004; Novotny et al., 2006) and directly associated with vegetation composition and structure (Seymour and Simmons, 2008; 
Lindenmayer et al., 2010). The difference in vegetation structure and food resources can attract bird species (Campbell et al., 2007; Holmes and Pitt, 2007) that prefers large areas of young sapling, regenerating and early successional vegetation (Costello et al., 2000; DeGraaf and Yamasaki, 2003).

Overall, the results of this study indicated that bird communities are dynamic and may changed in relation to recovery process after logging such as logging creates canopy gaps by removing trees, after logging during recovery process changes in vegetation structure may occurs over time which affects the avian richness and diversity through affecting food resources (Cambell et al. 2007) i.e., increased nest predation and brood parasitism (Rodewald, 2002; Thompson et al., 2002; Thompson and Burhans, 2003; Lemelin et al., 2007). During the recovery process many plant species become mature and start flowering and fruiting which attract insects (bees, wasps, butterflies, moths, beetles and flies) which is a major diet of avian species. Invertebrate communities of the tropical rain forest are highly diverse and their distribution and richness is associated with a diversity of vegetation structure and composition such as foliage, flowers, fruits, barks (Small and Pringle, 2010; Batista Matos et al., 2013; Peters et al., 2013). Silva and Brandao (2010) reported that invertebrate density strongly associated with vegetation structure and may vary at spatial scales of a few meters. Invertebrates are an important component of the food web in the forest ecosystem.

\section{CONCLUSION}

The findings of this study revealed that logging and recovery process may affects on avian distribution and diversity. However, these effects may vary from species to species. As the forest regenerates and recover the vegetation characteristics after logging may accommodate the higher bird species diversity and richness depending upon the rate of recovery process.

\section{ACKNOWLEDGEMENT}

The researchers would like to thank the Department of Wildlife and National Parks, Peninsular Malaysia (DWNP) for allowing us to conduct this research at different aged post-harvested hill dipterocarp rainforest habitats in Peninsular Malaysia. This research was funded by Research University Grants No: 03-01-111331RU.

\section{REFERENCES}

Arroyo-Rodriguez, V. and S. Mandujano, 2006. Forest fragmentation modifies habitat quality for Alouatta palliata. Int. J. Primatol., 27: 10791096. DOI: 10.1007/s10764-006-9061-0

Batista Matos, M.C., L. Sousa-Souto, R.S. Almeida and A.V. Teodoro, 2013. Contrasting patterns of species richness and composition of solitary wasps and bees (Insecta: Hymenoptera) according to land-use. Biotropica, 45: 73-79. DOI: 10.1111/j.1744-7429.2012.00886.x

Belisle, M., A. Desrochers and M.J. Fortin, 2001. Influence of forest cover on the movements of forest birds: A homing experiment. Ecology, 82: 1893-1904.

Brook, B.W., C.J. Bradshaw, L.P. Koh and N.S. Sodhi, 2006. Momentum drives the crash: Mass extinction in the tropics. Biotropica, 38: 302-305. DOI: 10.1111/j.1744-7429.2006.00141.x

Brook, B.W., N.S. Sodhi and P.K.L. Ng, 2003. Catastrophic extinctions follow deforestation in Singapore. Nature, 424: 420-423. DOI: 10.1038/nature01795

Campbell, S.P., J.W. Witham and M.L. Hunter Jr., 2007. Long-term effects of group selection timber harvesting on abundance of forest birds. Conserv. Biol., 21: 1218-1229. DOI: 10.1111/j.15231739.2007.00768.x

Cardillo, M., G.M. Mace, K.E. Jones, J. Bielby and A. Purvis et al., 2005. Multiple causes of high extinction risk in large mammal species. Science, 309: 1239-1241. DOI: 10.1126/science. 1116030

Castelletta, M., N.S. Sodhi and R. Subaraj, 2000. Heavy extinctions of forest avifauna in Singapore: Lessons for biodiversity conservation in Southeast Asia. Conserv. Bio., 14: 1870-1880. DOI: 10.1046/j.1523-1739.2000.99285.x

Castelletta, M., J.M. Thiollay and N.S. Sodhi, 2005. The effects of extreme forest fragmentation on the bird community of Singapore Island. Biol. Conser., 121: 135-155. DOI: 10.1016/j.biocon.2004.03.033

Chaves, Ó.M., K.E. Stoner and V. Arroyo-Rodríguez, 2012. Differences in diet between spider monkey groups living in forest fragments and continuous forest in Mexico. Biotropica, 44: 105-113. DOI: 10.1111/j.1744-7429.2011.00766.x 
Chazdon, R.L., S.G. Letcher, M. Van Breugel, M. Martínez-Ramos and B. Finegan et al., 2007. Rates of change in tree communities of secondary Neotropical forests following major disturbances. Philosophical Trans. Royal Society London B: Biol. Sci., 362: 273-289.

Clark, C.J., J.R. Poulsen, R. Malonga and P.W. Elkan, 2009. Logging concessions can extend the conservation estate for central African tropical forests. Conserv. Biol., 23: 1281-1293. DOI: 10.1111/j.1523-1739.2009.01243.x

Collins, A.C., 2008. The Taxonomic Status of Spider Monkeys in the Twenty-First Century. In: Spider Monkeys: Behavior, Ecology and Evolution of the Genus Ateles, Campbell, C.J. (Eds.), Cambridge University Press, New York, pp: 50-78.

Costello, C.A., M. Yamasaki, P.J. Pekins, W.B. Leak and C.D. Neefus, 2000. Songbird response to group selection harvests and clearcuts in a New Hampshire northern hardwood forest. Forest Ecol. Manage., 127: 41-54. DOI: 10.1016/S0378-1127(99)00131-0

Curran, L.M., S.N. Trigg, A.K. McDonald, D. Astiani and E. Kasischke et al., 2004. Lowland forest loss in protected areas of Indonesian Borneo. Science, 303: 1000-1003. DOI: 10.1126/science.1091714

Currie, D.J., G.G. Mittelbach, H.V. Cornell, R. Field, Guégan and J.R.G. Turner et al., 2004. Predictions and tests of climate-based hypotheses of broad-scale variation in taxonomic richness. Ecol. Lett., 7: 11211134. DOI: $10.1111 / \mathrm{j} .1461-0248.2004 .00671 . x$

DeFries, R., A. Hansen, A.C. Newton and M.C. Hansen, 2005. Increasing isolation of protected areas in tropical forests over the past twenty years. Ecol. Applic., 15: 19-26. DOI: 10.1890/03-5258

DeGraaf, R.M. and M. Yamasaki, 2003. Options for managing early successional forest and shrubland bird habitats in the north-eastern United States. Forest Ecol. Manage., 185: 179-191. DOI: 10.1016/S0378-1127(03)00254-8

Dent, D.H. and S.J. Wright, 2009. The future of tropical species in secondary forests: A quantitative view. Biol. Conserv., 142: 2833-2843. DOI: 10.1016/j.biocon.2009.05.035

Doyon, F., D. Gagnon and J.F. Giroux, 2005. Effects of strip and single tree selection cutting on birds and their habitat in a South-Western Quebec Northern Hardwood forest. Forest Ecol. Manag., 209: 101115. DOI: 10.1016/j.foreco.2005.01.005
Echeverria, C., A.C. Newton, A. Lara, J.M.R. Benayas and D.A. Coomes, 2007. Impact of forest fragmentation on species composition and forest structure in the temperate landscape of southern Chile. Global Eco. Biogeogr., 16: 426-439. DOI: 10.1111/j.1466-9238/2007.0031/x

Edwards, D.P., T.H. Larsen, T.D.S. Docherty, F.A. Ansell and D.S. Wilcove et al., 2011. Degraded lands worth protecting: The biological importance of Southeast Asia's repeatedly logged forests. Proceedings of the Royal Society Bulletin. (RSB'11), Cambridge University Press, New York, pp: 82-90.

Ellwood, M.D.F. and W.A. Foster, 2004. Doubling the estimate of invertebrate biomass in a rainforest canopy. Nature, 429: 549-551. DOI: 10.1038 /nature 02560

Ellwood, M.D.F., D.T. Jones and W.A. Foster, 2002. Canopy ferns in lowland dipterocarp forest support a prolific abundance of ants, termites and other invertebrates. Biotropica, 34: 575-583.

Fahrig, L., 2003. Effects of habitat fragmentation on biodiversity. Annual Rev. Eco. Syst., 34: 487-515.

Fisher, B., D.P. Edwards, T.H. Larsen, F.A. Ansell and D.S. Wilcove et al., 2011. Cost-effective conservation: Calculating biodiversity and logging tradeoffs in Southeast Asia. Conserv. Lett., 84: 443450. DOI: 10.1111/j.1755-263X.2011.00198.x

Fitzherbert, E.B., M.J. Struebig, A. Morel, F. Danielsen and B. Phalan et al., 2008. How will oil palm expansion affect biodiversity? Trends Ecol. Evolut., 23: 538-545. DOI: 10.1016/j.tree.2008.06.012

Gill, F.G., 2006. Ornithiology. 3rd Edn., Freeman, W.H. and Company. ISBN-10: 0716749831. Pp: 720.

Gram, W.K., P.A. Porneluzi, R.L. Clawson, J. Faaborg and S.C. Richer, 2003. Effects of experimental forest management on density and nesting success of bird species in Missouri Ozark forest. Conserv. Bio., 17: 1324-1337. DOI: 10.1046/j.15231739.2003.02171.x

Henderson, P.A. and R.M.H. Seaby, 2007. Community Analysis Package 4.0, Pisces Conservation Ltd, Lymington, UK.

Hill, J.L. and P.J. Curran, 2003. Area, shape and isolation of tropical forest fragments: Effects on tree species diversity and implications for conservation. J. Bio., 30: 1391-1403.

Holmes, R.T. and D.G. Pitt, 2007. Response of bird communities to selection harvesting in a northern tolerant hardwood forest. Forest Ecol. Manage., 238: 280-292. DOI: 10.1016/j.foreco.2006.10.022 
Ishikawa, Y., T. Sakamoto and K. Mizuhara, 2003. Effect of density riparian vegetation on effective tractive force. J. Forest Res., 8: 235-246. DOI: 10.1007/s10310-003-0032-4

Jackson, S.M., T.S. Fredericksen and J.R. Malcolm, 2002. Area disturbed and residual stand damage following logging in a Bolivian tropical forest. Forest Ecol. Manag., 166: 271-283. DOI: 10.1016/S0378-1127(01)00681-8

Johns, A.D., 1988. Effects of selective timber extraction on rain forest structure and composition and some consequences for frugivores and folivores. Bio., 20: 31-37.

Johnson, M.D. and T.W. Sherry, 2001. Effects of food availability on the distribution of migratory warblers among habitats in Jamaica. J. Anim, Ecol., 70: 546560. DOI: 10.1046/j.1365-2656.2001.00522.x

King, D.I. and R.M. Degraaf, 2004. Effects of groupselection opening size on the distribution and reproductive success of an early-successional shrubland birds. Forest Ecol. Manage., 190: 179185. DOI: $10.1016 /$ j.foreco.2003.10.012

Kinnard, M.F., T.G. O'Brien and S. Suryadi, 1996. Population fluctuation in Sulawesi Red-knobbed Hornbills: Tracking figs in space and time. Auk, 113: 431-440. DOI: $10.2307 / 4088909$

Koh, L.P., N.S. Sodhi and B.W. Brook, 2004. Ecological correlates of extinction proneness in tropical butterflies. Conserv. Biol., 18: 1571-1578. DOI: 10.1111/j.1523-1739.2004.00468.x

Laurance, W.F., 1999. Reflections on the tropical deforestation crisis. Biol. Conserv., 91: 109-117. DOI: 10.1016/S0006-3207(99)00088-9

Lemelin, L.V., L. Imbeau, M. Darveau and D. Bordage, 2007. Local, short-term effects of forest harvesting on breeding waterfowl and Common Loon in forestdominant landscapes of Quebec. Avian Conserv. Ecol., 2: pp: 10.

Lindenmayer, D.B., J.T. Wood, L. McBurney, D. Michael, M. Crane, C. MacGregor andR.M. Drake, 2010. Comparing bird species richness and assemblage composition between montane ash eucalypt forest and cool temperate rainforests-an empirical study from Victoria, south-eastern Australia. $\quad$ Emu, 110: 109-117. DOI: 10.1071/MU09074

Malizia, L.R., 2001. Seasonal fluctuations of birds, fruits and flowers in a subtropical forest of Argentina. Condor, 103: 45-61. DOI: 10.1650/00105422(2001)103[0045:SFOBFA]2.0.CO;2
Marquez, A.L., R. Real and J.M. Vargas, 2004. Dependence of broad-scale geographical variation in fleshy-fruited plant species richness on disperser bird species richness. Global Ecol. Biol., 13: 295304. DOI: $10.1111 / \mathrm{j} .1466-822 X .2004 .00100 . x$

McCarthy, B., 2012. The effects of logging and fragmentation on bird diversity. Plymo. Stu. Sci., 5: 558-568.

McGraw-Hill, C., 2008. Statistix $8.1 \quad$ (Analytical Software, Tallahassee, Florida). Maurice/Thomas text.

McShea, W.J. and J.H. Rappole, 2000. Managing the abundance and diversity of breeding bird populations through manipulation of deer populations. Conserv. Biol., 14: 1161-1170. DOI: 10.1046/j.1523-1739.2000.99210.x

Moegenburg, S.M. and D.J. Levey, 2003. Do frugivore respond to fruit harvest? An experimental study of short-term response. Ecology, 84: 2600-2612. DOI: 10.1890/02-0063

Moorman, C.E., D.C. Guynn Jr. and J.C. Kilgo, 2002. Hooded warbler nesting success adjacent to groupselection and clearcut edges in a southeastern bottomland form. Condor, 104: 366-377. DOI: $10.1650 / 0010-$ 5422(2002)104[0366:HWNSAT]2.0.CO;2

Novotny, V., P. Drozd, S.E. Miller, M. Kulfan and G.D. Weiblen et al., 2006. Why are there so many species of herbivorous insects in tropical rainforests. Science, 313: 1115-1118. DOI: $10.1126 /$ science. 1129237

Pers, C.M., 2000. Identifying keystone plant resources from tropical forests: The case of gums from Parkia pods. J. Tropical Ecol., 16: 287-317. DOI: $10.1017 / \mathrm{S} 0266467400001413$

Peters, M.K., G. Fischer, F. Hita Garcia, T. Lung and J.W. Wägele et al., 2013. Spatial variation in army ant swarm raiding and its potential effect on biodiversity. Biotropica, 45: 54-62. DOI: 10.1111/j.1744-7429.2012.00890.x

Potts, K.B., 2011. The Long-term Impact of Timber Harvesting on the Resource Base of Chimpanzees in Kibale National Park, Uganda. Biotropica, 43: 256264. DOI: 10.1111/j.1744-7429.2010.00671.x

Rajpar, M.N. and M. Zakaria, 2010. Bird species composition and feeding guilds based on point count and mist netting methods at the Paya Indah Wetland Reserve, Peninsular Malaysia. Trop. Life Sci. Res., 21: 7-26. PMID: 24575196 
Rajpar, M.N. and M. Zakaria, 2012. Avian community parameters of a freshwater wetland ecosystem in Peninsular Malaysia. Asia Life Sci., 21: 409-428.

Ralph, C.J. and E.H. Dunn, 2004. Monitoring Bird Populations Using Mist Nets. In: Cadmus Communication Corporation, Ephrata, Pennsylvania, Rotenberry, J.T. (Ed.)., Studies in Avian Biology, pp: 1-6.

Renton, K., 2001. Lilac-crowned Parrot diet and food resources availability: Resource tracking by a parrot seed predator. Condor, 103: 62-69. DOI: 10.1650/00105422(2001)103[0062:LCPDAF]2.0.CO;2

Robbins, L.L., Y. Tao and C. A. Evans, 1997. Temporal and spatial distribution of whitings on Great Bahama Bank and a new lime mud budget. Geology, 25: 947-950.

Robinson, W.D. and S.K. Robinson, 1999. Effects of selective logging on forest bird populations in a fragmented landscape. Conserv. Biol., 13: 58-66. DOI: $10.1046 /$ j.1523-1739.1999.97226.x

Robson, C., 2002. Real World Research: A Resource for Social Scientists and Practitioner Research. 1st Edn., Blackwell Publishing, Malden.

Rodewald, A.D., 2002. Nest predation in forested regions: Landscape and edge effects. J. Wildlife Manag., 66: 634-640. DOI: 10.2307/3803130

Sehgal, R.N.M., 2010. Deforestation and avian infectious diseases. J. Exp. Biol., 213: 955-960. DOI: 10.1242/jeb.037663

Seymour, C.L. and R.E. Simmons, 2008. Can severely fragmented patches of riparian vegetation still be important for arid-land bird diversity? J. Arid Environ., 72: 2275-2281. DOI: 10.1016/j.jaridenv.2008.07.014
Sherman, P.T. and P.K. Eason, 1998. Size determinants in territories with inflexible boundaries: Manipulation experiments on White-winged trumpeters territories. Ecology, 79: 1147-1159.

Silva, R.R. and C.R.F. Brandao, 2010. Morphological patterns and community organization in leaf-litter ant assemblages. Ecol. Monograph, 80: 107-124. DOI: $10.1890 / 08-1298.1$

Small, G.E. and C.M. Pringle, 2010. Deviation from strict homeostasis across multiple trophic levels in an invertebrate consumer assemblage exposed to high chronic phosphorus enrichment in a Neotropical stream. Oecologia, 162: 581-590. DOI: 10.1007/s00442-009-1489-4

Sodhi, N.S. and B.W. Brook, 2006. Southeast Asian Biodiversity in Crisis. 1st Edn., Cambridge University Press, Cambridge.

Thompson, F.R., T.M. Donovan, R.M. DeGraaf, J. Faaborg and S.K. Robinson et al., 2002. A multiscale perspective of the effects of forest fragmentation on birds in eastern forests. Stud. Avian Biol., 25: 8-19.

Thompson, F.R. and D.E. Burhans, 2003. Predation of Songbird Nests Differs by Predator and between Field and Forest Habitats. J. Wildlife Manage., 67: 408-416.

Wright, S.J., C. Carrasco, O. Calderon and S. Paton, 1999. The ElNino Southern Oscillation, variable fruit production and famine in a tropical forest. Ecology, 80: 1632-1647. 